\title{
Spin dynamics and orbital-antiphase pairing symmetry in iron-based superconductors
}

\author{
Z. P. Yin ${ }^{\star}$, K. Haule and G. Kotliar
}

The symmetry of the wavefunction describing the Cooper pairs is one of the most fundamental quantities in a superconductor, but for iron-based superconductors it has proved to be problematic to determine, owing to their complex multi-band nature $^{1-3}$. Here we use a first-principles many-body method, including the two-particle vertex function, to study the spin dynamics and the superconducting pairing symmetry of a large number of iron-based compounds. Our results show that these high-temperature superconductors have both dispersive high-energy and strong low-energy commensurate or nearly commensurate spin excitations, which play a dominant role in Cooper pairing. We find three closely competing types of pairing symmetries, which take a very simple form in the space of active iron $3 d$ orbitals, and differ only in the relative quantum mechanical phase of the $x z, y z$ and $x y$ orbital components of the Cooper pair wavefunction. The extensively discussed $s^{+-}$ symmetry appears when contributions from all orbitals have equal sign, whereas a novel orbital-antiphase $s^{+-}$symmetry emerges when the $x y$ orbital has an opposite sign to the $x z$ and $y z$ orbitals. This orbital-antiphase pairing symmetry agrees well with the angular variation of the superconducting gaps in LiFeAs (refs 4,5).

The spin and the multi-orbital dynamics of iron-based superconductors are believed to play an essential role in the mechanism of superconductivity ${ }^{6}$, but a realistic modelling of magnetic excitations, and a clear physical picture for their variation across different families of iron superconductors, is currently lacking. The Cooper pairs are locked into singlets, but the orbital structure of the superconducting order parameter can be material dependent, and its connection to orbital and spin excitations is an open problem.

The charge dynamics of the iron-based superconductors is controlled by the strong Hund's coupling on the iron site $e^{7,8}$, which requires a theoretical approach that simultaneously treats the itinerancy of the electrons and Hund's interaction on an equal footing. Using non-perturbative many-body method and $a b$ initio-determined two-particle scattering amplitude (the twoparticle vertex function), we are able to accurately describe the spin dynamics and symmetry of the superconducting order parameter, and we will show that Hund's rule coupling and orbital blocking ${ }^{9}$ play a crucial role in the superconductivity of iron superconductors.

All iron-based superconductors contain the same basic motiflayers of iron atoms tetrahedrally coordinated by pnictogen or chalcogen atoms-but their spin excitation spectra varies greatly among compounds. In Fig. 1 we plot the dynamic spin structure factor $\mathrm{S}(q, \omega)=\chi^{\prime \prime}(q, \omega) /\left(1-\exp \left(-\hbar \omega / k_{\mathrm{B}} T\right)\right)$ in the paramagnetic state for several classes of iron compounds along the high-symmetry momentum path in the first Brillouin zone of the single-iron unit cell. Here, the momentum transfer is labelled using the same convention as used in neutron scattering experiments ${ }^{10}$. We overlay the neutron scattering data ${ }^{10-13}$ for some compounds where experimental results are available, to show the good agreement between theory and experiment. We computed the magnetic excitations in the paramagnetic state, at temperatures above the spin density wave (SDW) transition (even for compounds that have a magnetically ordered ground state) and compared them with experimental results in the paramagnetic state.

The bandwidth of the spin excitations, defined as the difference in the excitation energy at the two momentum points $q=(1,0)$ and $q=(1,1)$, which are the minimum and the maximum of the dispersion curve respectively, varies substantially throughout $\mathrm{Fe}$ compounds. In strong-coupling theories, this spin bandwidth is related to the spin-exchange constant $J$, and is therefore inversely proportional to the interaction strength $\left(J \propto t^{2} / U\right)$. We find that in the Hund's metals ${ }^{9}$, the bandwidth also increases with decreasing correlation strength, as determined by the degree of mass renormalization. The strength of the electronic correlations is tuned by the iron $3 d$ occupancy $^{14}$ and the Fe-pnictogen distance ${ }^{9}$.

The phosphorus compounds (Fig. 1a-c) exhibit the largest spin-wave bandwidth (of the order of $0.6 \mathrm{eV}-0.45 \mathrm{eV}$ ), which is a consequence of their most itinerant nature among these compounds. The mass enhancement due to correlations is increased in arsenides, and even more in the chalcogenides ${ }^{9}$, hence the spin-wave bandwidth is progressively reduced to $0.3-0.2 \mathrm{eV}$ in Fig. 1d-f, and $0.15-0.10 \mathrm{eV}$ in Fig. 1g-h. The intensity of the spin excitations is proportional to the size of the fluctuating moment in this energy range, which roughly correlates with the strength of correlations, hence phosphorus compounds show the weakest $(\mathrm{Max}=4)$ and FeTe shows the strongest $(\mathrm{Max}=20)$ intensity.

The low-energy spin excitations are much more sensitive to the details of both the band structure and the two-particle vertex function, hence the trend across different compounds can not be guessed from either the correlation strength or from the band structure. In Fig. 2 we show $S(q, \omega)$ for the same compounds as in Fig. 1, but we take a different cut in momentum-energy space, keeping the energy fixed at $\omega=5 \mathrm{meV}$, and changing the momentum in the two-dimensional momentum plane $(H, K)$. As is clear from Figs $1 \mathrm{a}-\mathrm{c}$ and $2 \mathrm{a}-\mathrm{c}$, the low-energy spin excitations are extremely weak $(\operatorname{Max} \approx 1)$ in phosphorus compounds and the spin excitations at the SDW ordering vector $(1,0)$ is comparable to its value at the ferromagnetic ordering vector $(0,0)$. In strong contrast, the low-energy spin excitations are very strong in arsenides (Fig. 2d-g) and are concentrated solely at the commensurate wavevector $(H, K)=(1,0)$. This is the ordering wavevector of the SDW magnetic state, which is the ground state for these parent compounds, except the superconducting LiFeAs $\left(T_{C}=18 \mathrm{~K}\right)$. 
a

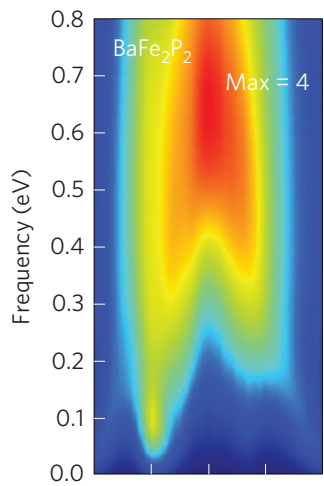

f

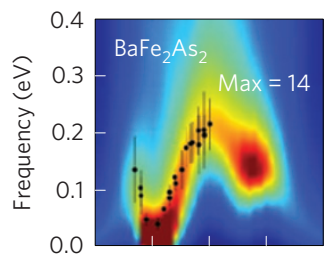

k

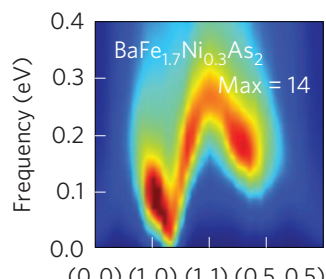

$(H, K)$ (r.l.u.)
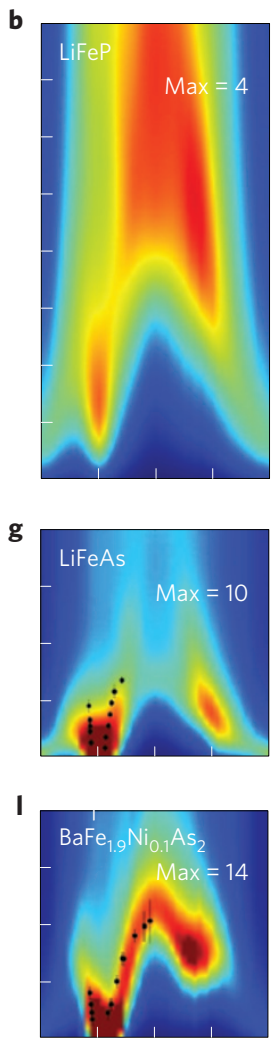

$(0,0)(1,0)(1,1)(0.5,0.5)$

$(H, K)$ (r.l.u.)
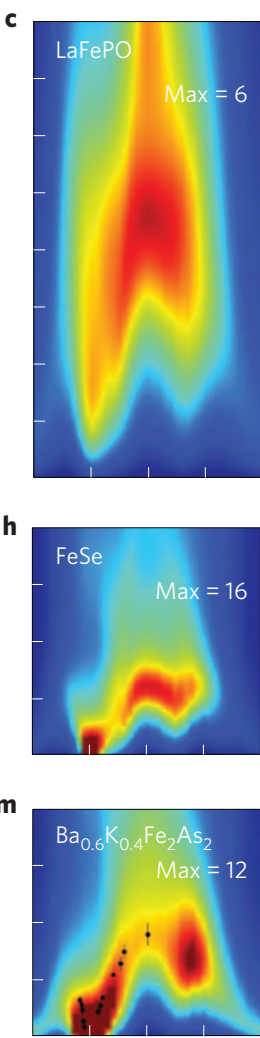

$(0,0)(1,0)(1,1)(0.5,0.5)$

$(H, K)$ (r.l.u.)
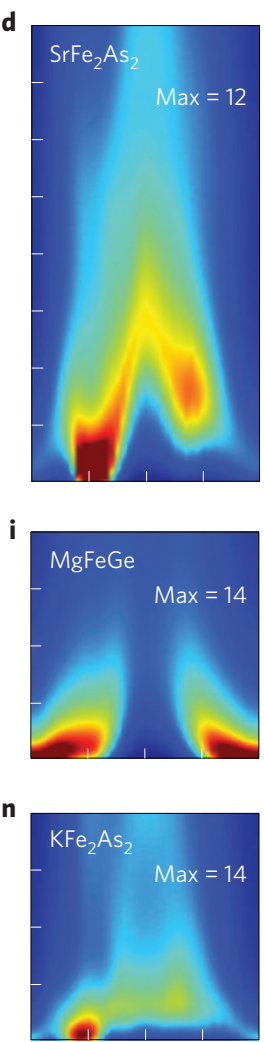

$(0,0)(1,0)(1,1)(0.5,0.5)$

$(H, K)$ (r.l.u.)
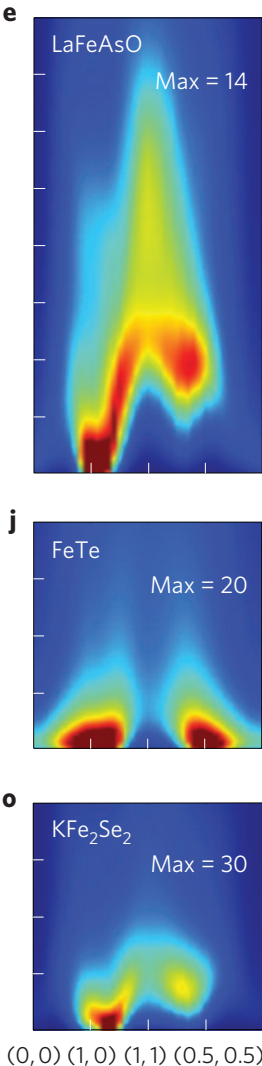

$(H, K)$ (r.l.u.)

Figure 1 | Dynamic spin structure factor $S(q, \omega)$ in iron pnictides, chalcogenides and MgFeGe. $S(q, \omega)$ is plotted along the high-symmetry path $(H, K, L=1)$ in the first Brillouin zone of the single-iron unit cell. The intensity varies substantially across these compounds, hence the maximum value of the intensity was adjusted to emphasize the dispersion most clearly. The maximum value of the intensity in each compound is shown in the top-right corner. The colour coding corresponds to the theoretical calculations for (a) $\mathrm{BaFe}_{2} \mathrm{P}_{2}\left(T_{C}^{\mathrm{max}}<2 \mathrm{~K}\right) ;(\mathbf{b}) \mathrm{LiFeP}\left(T_{C}=6 \mathrm{~K}\right) ;(\mathbf{c}) \mathrm{LaFePO}_{(}\left(T_{C}=7 \mathrm{~K}\right) ;(\mathbf{d}) \mathrm{SrFe}_{2} \mathrm{As}_{2}\left(T_{C}^{\mathrm{max}}=37 \mathrm{~K}\right)$; (e) $\mathrm{LaFeAsO}\left(T_{C}^{\max }=43 \mathrm{~K}\right) ;(\mathbf{f}) \mathrm{BaFe}_{2} \mathrm{As}_{2}\left(T_{C}^{\max }=39 \mathrm{~K}\right) ;(\mathbf{g}) \mathrm{LiFeAs}\left(T_{C}=18 \mathrm{~K}\right) ;(\mathbf{h}) \mathrm{FeSe}\left(T_{C}^{\mathrm{max}}=37 \mathrm{~K}\right) ;(\mathbf{i}) \mathrm{MgFeGe}\left(T_{C}^{\mathrm{max}}=0 \mathrm{~K}\right) ;(\mathbf{j}) \mathrm{FeTe}\left(T_{C}^{\mathrm{max}}=0 \mathrm{~K}\right)$; (k) $\mathrm{BaFe}_{1.7} \mathrm{Ni}_{0.3} \mathrm{As}_{2}\left(T_{C}<2 \mathrm{~K}\right) ;(\mathbf{I}) \mathrm{BaFe}_{1.9} \mathrm{Ni}_{0.1} \mathrm{As}_{2}\left(T_{C}=20 \mathrm{~K}\right) ;(\mathbf{m}) \mathrm{Ba}_{0.6} \mathrm{~K}_{0.4} \mathrm{Fe}_{2} \mathrm{As}_{2}\left(T_{C}=39 \mathrm{~K}\right) ;(\mathbf{n}) \mathrm{KFe}_{2} \mathrm{As}_{2}\left(T_{C}=3.5 \mathrm{~K}\right) ;(\mathbf{o}) \mathrm{KFe}_{2} \mathrm{Se}_{2} . \mathrm{Th}$ experimental data are shown as black dots with error bars in $\mathbf{f}, \mathbf{g}, \mathbf{I}$ and $\mathbf{m}$, digitized from refs 10-13. r.l.u., reciprocal lattice units.

When doped, all compounds in Fig. 2d-f are high-temperature superconductors $\left(T_{C} \approx 37 \mathrm{~K}-39 \mathrm{~K}\right)$. Similarly chalcogenide FeSe (Fig. 1h), which becomes superconducting at $T_{C}=36 \mathrm{~K}$ under modest pressure $p=4 \mathrm{GPa}$ (ref. 15), has a similar low-energy spin response to the arsenide superconductors. The pronounced difference in the low-energy intensity of the spin excitations between the phosphorus and the arsenide compounds is due both to the reduced correlation strength in phosphorus compounds ${ }^{9}$ (Supplementary Methods) and the difference in the orbital content of the Fermi surface discussed in ref. 16.

$\mathrm{MgFeGe}$ is a non-superconducting compound with a band structure very similar to LiFeAs, and serves as a strong test of a theoretical description of this class of compounds. Within the random phase approximation, the two compounds have very similar spin excitations, with low-energy maximum intensity at $q=(1,0)$ (ref. 17). The inclusion of both strong electronic correlations and the realistic two-particle vertex function, as done in this study, identifies clear differences between these two compounds. A broad maximum appears in the response of $\mathrm{MgFeGe}$ at $q=(0,0)$ (Fig. 2i), hence spin fluctuations are ferromagnetic, in agreement with calculations of ref. 18 showing a stable ferromagnetic ground state. Finally, FeTe also has much broader spin excitations, covering a large part of the Brillouin zone (see Fig. 2j), and shows two competing excitations at $q=(1,0)$ and $q=(0.5,0.5)$-the latter corresponding to the ordering wavevector of the low-temperature antiferromagnetic state of $\mathrm{Fe}_{1.07} \mathrm{Te}$ (ref. 19).
The common theme among the high-temperature superconductors (Figs $1 \mathrm{~d}-\mathrm{h}$ and $2 \mathrm{~d}-\mathrm{h}$ ) is thus the existence of welldefined high-energy dispersive spin excitations with spin-wave bandwidths between 0.1 and $0.35 \mathrm{eV}$ and, most importantly, very well developed commensurate (or nearly commensurate) lowenergy spin excitations at wavevector $q=(1,0)$, and equivalently $q=(0,1)$, consistent with the theory of spin-fluctuation-mediated superconductivity ${ }^{20}$. The pnictide parent compounds $\mathrm{SrFe}_{2} \mathrm{As}_{2}$, $\mathrm{LaFeAsO}$ and $\mathrm{BaFe}_{2} \mathrm{As}_{2}$ have strong low-energy spin excitations centred exactly at $q=(1,0)$, whereas in LiFeAs and FeSe the spin excitations are peaked slightly away from this commensurate wavevector. Consequently, the former three compounds have an antiferromagnetic ground state, whereas the latter two are superconducting. In the former, electron or hole doping is needed to suppress the long-range magnetic order, and to stabilize the competing superconducting state. In Figs $1 \mathrm{f}, \mathrm{k}-\mathrm{n}$ and $2 \mathrm{f}, \mathrm{k}-\mathrm{n}$ we illustrate the doping dependence of the spin excitation spectrum on the examples of electron-doped and hole-doped $\mathrm{BaFe}_{2} \mathrm{As}_{2}$ - that is, $\mathrm{BaFe}_{2-x} \mathrm{Ni}_{x} \mathrm{As}_{2}$ and $\mathrm{Ba}_{1-x} \mathrm{~K}_{x} \mathrm{Fe}_{2} \mathrm{As}_{2}$, respectively. The electron doping slightly increases the spin-wave bandwidth (comparing Fig. If with $\mathrm{k}$ ), whereas the hole doping markedly reduces the spin-wave bandwidth from $\sim 0.2 \mathrm{eV}$ to $\sim 0.05 \mathrm{eV}$ in overdoped $\mathrm{KFe}_{2} \mathrm{As}_{2}$ (ref. 13; Fig. 1n). The low-energy spin excitations in the electron-overdoped $\mathrm{BaFe}_{1.7} \mathrm{Ni}_{0.3} \mathrm{As}_{2}$ become very weak and strongly incommensurate ${ }^{13}$, with a peak centred at $q=(1.0,0.35)$ (see Fig. 2k). Similarly, on the hole-overdoped side in $\mathrm{KFe}_{2} \mathrm{As}_{2}$, the low-energy spectrum 

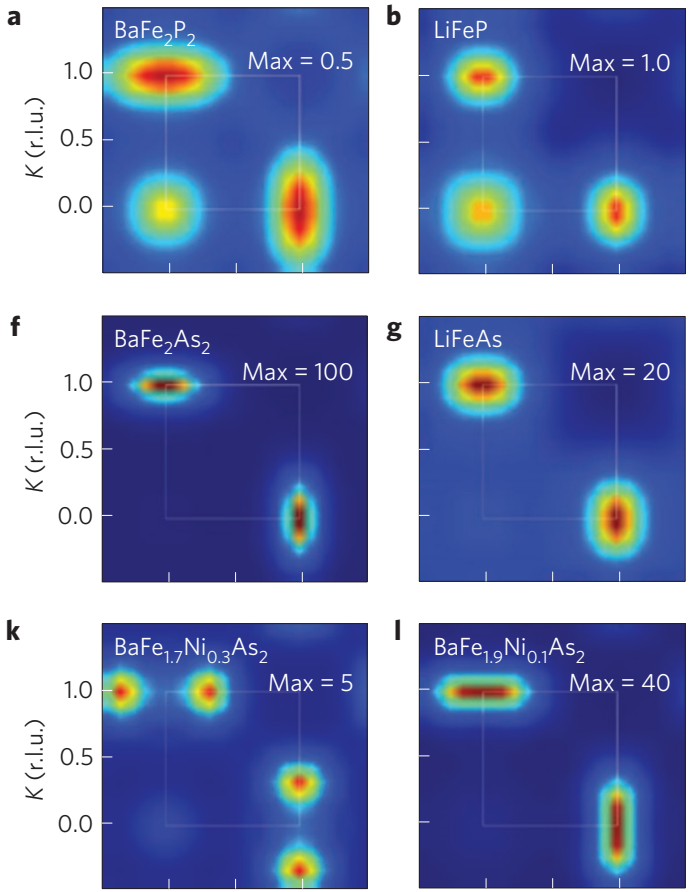

$\begin{array}{lll}0.0 & 0.5 & 1.0\end{array}$ $H$ (r.l.u.)

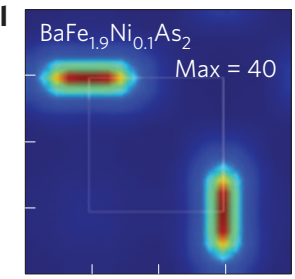

$\begin{array}{lll}0.0 & 0.5 & 1.0\end{array}$ $H$ (r.l.u.)
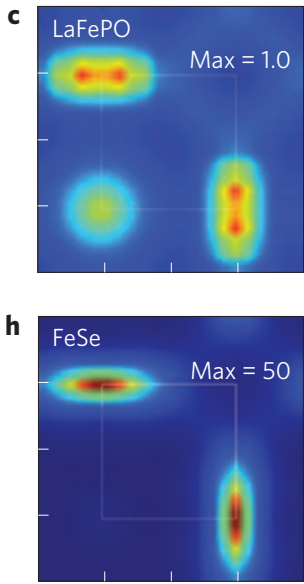

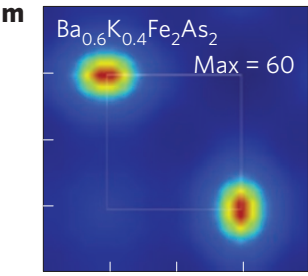

$0.0 \quad 0.5 \quad 1.0$ $H$ (r.l.u.)
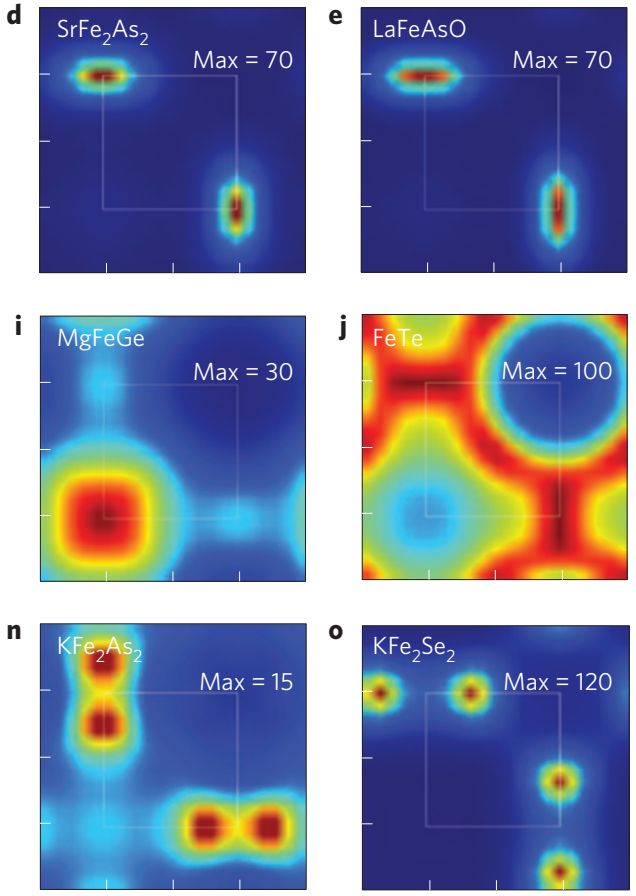

$\begin{array}{lll}0.0 & 0.5 & 1.0\end{array}$ $H$ (r.l.u.)

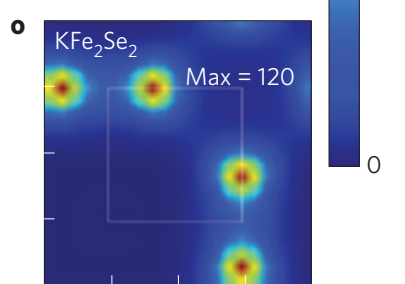

$0.0 \quad 0.5 \quad 1.0$ $H$ (r.l.u.)

Figure 2 | Dynamic spin structure factor $\mathbf{S}(q, \omega)$ in iron pnictides, chalcogenides and $\mathbf{M g F e G e . ~} S(q, \omega)$ is plotted in the $2 \mathrm{D}$ plane $(H, K)$ at constant $\omega=5$ meV for the same materials as in Fig. 1. The maximum intensity scale for each compound is marked as a number in the top-right corner of each subplot. The momentum dependence in the $k_{z}$ direction is weak in most compounds, hence we show only the cut at $L=1$. In $M g F e G e$ and phosphorus compounds, we instead show the $L=0$ plane to emphasize the tendency towards ferromagnetism in these compounds. r.l.u., reciprocal lattice units.

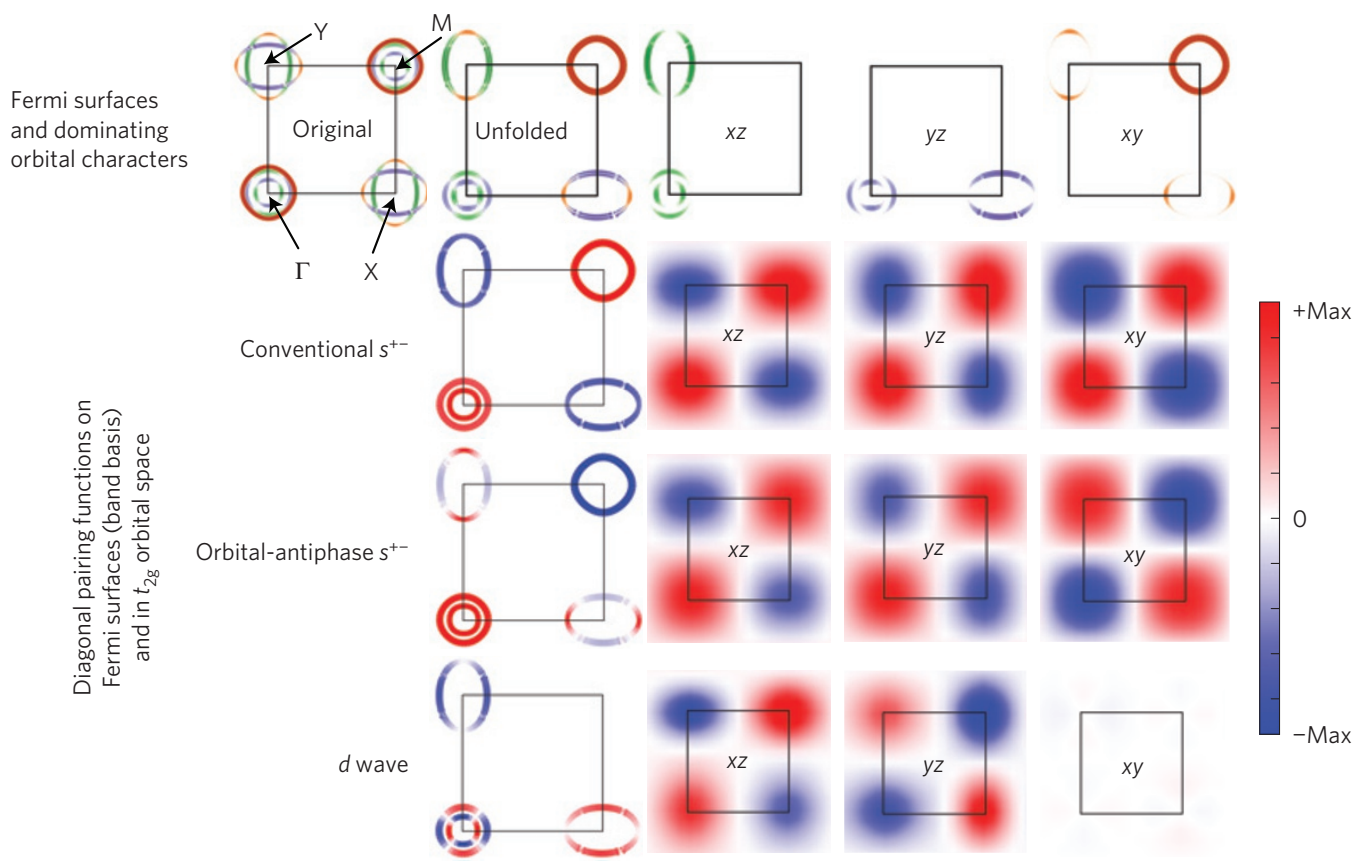

Figure 3 | Fermi surfaces, pairing symmetries and the basic building blocks. Top row: the original and unfolded two-dimensional Fermi surfaces in the $\Gamma$ plane for the representative compound LaFeAsO in the paramagnetic state, shown in the first Brillouin zone of the single-iron unit cell. On the top right, the Fermi surfaces are further decomposed into the dominating Fe- $t_{2 g}(x z, y z$, and $x y)$ characters. The next three rows, from top to bottom, show respectively the conventional $s^{+-}$, the orbital-antiphase $s^{+-}$and the $d$-wave pairing symmetries of the superconducting order parameter. The left column shows the Fermi surfaces, coloured with the strength of the diagonal order parameter $\Delta_{i i}(\mathbf{k})=\left\langle c_{k \uparrow, i}^{+} c_{-k \downarrow, i}^{+}\right\rangle$in the band basis ( $i$ is the band index), while the right columns decompose the order parameter in orbital space-that is, $\Delta_{\alpha \alpha}(\mathbf{k})=\left\langle c_{k \uparrow, \alpha}^{+} c_{-k \downarrow, \alpha}^{+}\right\rangle$( $\alpha$ runs over Fe- $t_{2 g}$ orbitals: $x z, y z$, and $x y$ ). Note that the order parameter is not constant on each of the Fermi surfaces in the conventional $s^{+-}$state. Whereas the diagonal order parameter in the band basis has nodes on the electron Fermi surfaces in the orbital-antiphase $s^{+-}$state, the spectral superconducting gap can be nodeless as a result of interband pairing (see text and Fig. 4). 
a

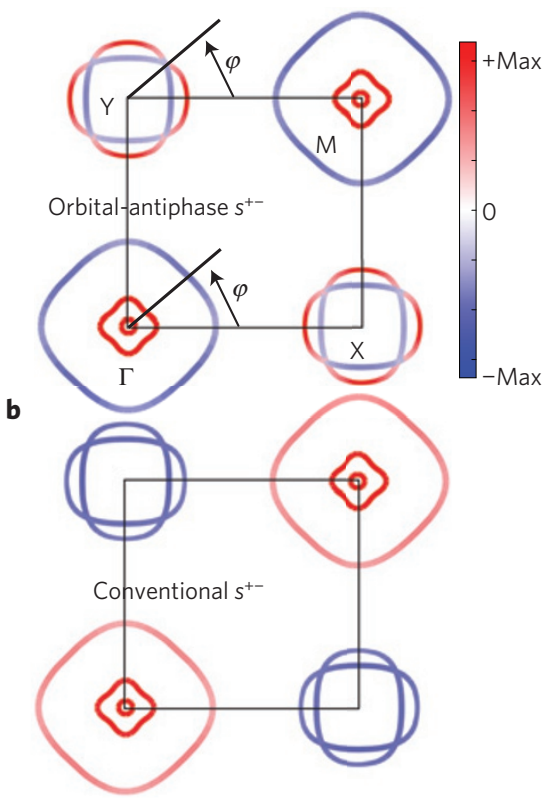

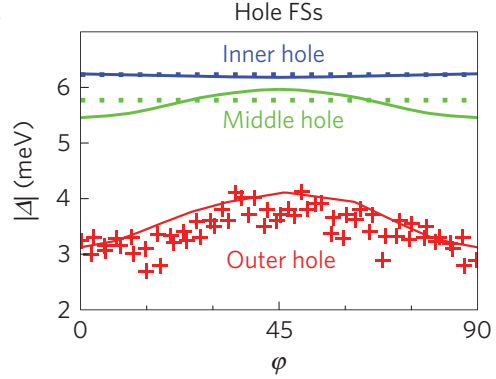

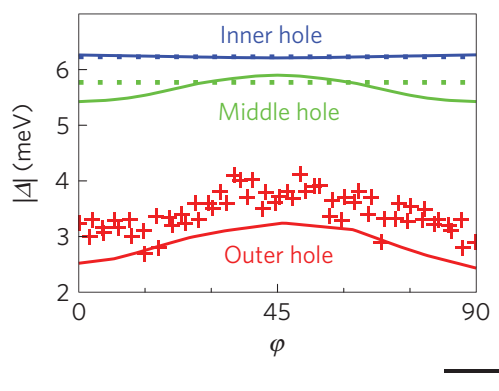

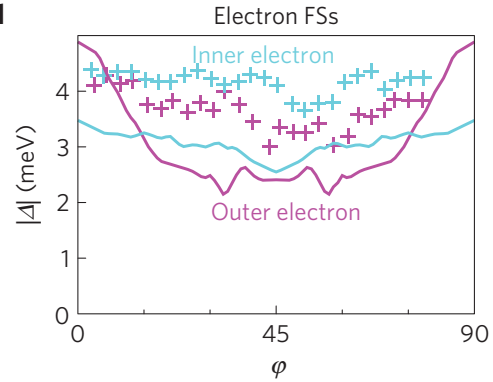

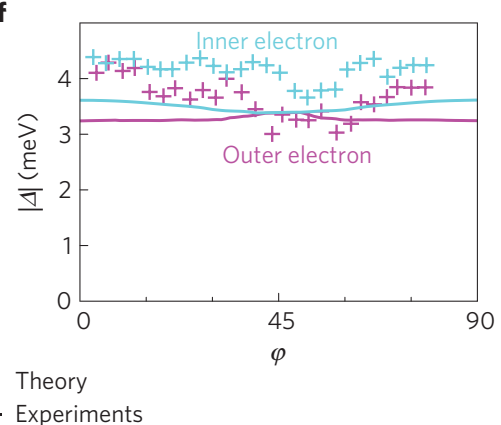

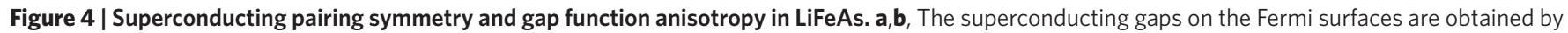

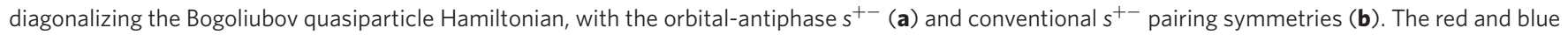
colours denote the different signs of the superconducting gaps. c-f, Angular dependences of the superconducting gaps for the orbital-antiphase $s^{+-}$(c,d) and conventional $s^{+-}(\mathbf{e}, \mathbf{f})$ states on the three-hole Fermi surfaces $(\mathbf{c}, \mathbf{e})$ and the two electron Fermi surfaces (d,f), respectively. The solid lines correspond to theoretical results and the symbols denote the experimental measurements from ref. 4 . Note that we rescaled the whole gap function such that the computed superconducting gap on the inner hole pocket matches the experimental value ${ }^{4}$.

is suppressed (maximum intensity in Fig. $2 \mathrm{n}$ is 15 , compared to 100 in the parent compound), and the main excitation peak moves to an incommensurate wavevector $q=(0.75,0)$ in agreement with experiments ${ }^{13,21}$. The optimally doped compounds (Fig. 11,m) have high-energy spin excitations very similar to the parent compound, whereas the low-energy excitations are slightly reduced and broadened in momentum space (Fig. 2l,m), to suppress the long-range magnetic order of the parent compound. This is very similar to the spectrum of LiFeAs and FeSe, both of which have a superconducting ground state. From these plots, we can deduce that nearly commensurate or commensurate spin excitations at $q=(1,0)$, with some finite width in momentum space to reduce the tendency towards the long-range magnetic order, are favourable for superconductivity.

Turning to $\mathrm{KFe}_{2} \mathrm{Se}_{2}$, Figs 10 and 2o indicate strong low-energy spin excitations peaked around $q=(1,0.4)$. Vacancies in the $\mathrm{K}$ site, which reduce the effective electron doping, can move the peak towards $q=(1,0)$ and favour superconductivity. On the other hand, vacancies in the Fe sites can move the peak to $q=(0.6,0.2)$ to induce novel magnetism in $\mathrm{K}_{0.8} \mathrm{Fe}_{1.6} \mathrm{Se}_{2}$ (ref. 22).

Whereas the dispersion of the dynamic spin structure factor $S(q, \omega)$ and the strength of the low-energy spin excitations correlate with experimental $T_{C}$ across many families of iron superconductors, the superconducting pairing symmetry and the variation of the superconducting gaps on different Fermi surfaces cannot be extracted from the spin dynamics alone. To make further progress on these issues, we computed the complete two-particle scattering amplitude in the particle-particle channel and determined the superconducting pairing function (Methods).

In compounds with strong low-energy (nearly) commensurate spin excitations, such as $\mathrm{SrFe}_{2} \mathrm{As}_{2}, \mathrm{LaFeAsO}, \mathrm{BaFe}_{2} \mathrm{As}_{2}, \mathrm{LiFeAs}$, FeSe, $\mathrm{BaFe}_{1.9} \mathrm{Ni}_{0.1} \mathrm{As}_{2}$ and $\mathrm{Ba}_{0.6} \mathrm{~K}_{0.4} \mathrm{Fe}_{2} \mathrm{As}_{2}$, the eigenvalue problem that determines the pairing function has three, almost degenerate, leading eigensolutions (with eigenvalues differing by only a few per cent). The largest eigenvalue of the pairing equations generates a pole in the particle-particle scattering process, and consequently determines the wavefunction of the Cooper pair. The corresponding three eigenvectors, which are proportional to the superconducting order parameter $\Delta_{\alpha \beta}(\mathbf{k})(\alpha, \beta$ are orbital indices of the iron $3 d$ orbitals), have a surprisingly simple form in the orbital space. The momentum dependence of the order parameter is very close to $\cos \left(k_{x}\right) \cos \left(k_{y}\right)$ and $\Delta_{\alpha \beta}(\mathbf{k})$ is almost diagonal in the orbital indices. The numerical solutions can therefore be approximated as

$$
\Delta_{\alpha \beta}(\mathbf{k}) \approx \delta_{\alpha \beta} \Delta_{\alpha} \cos \left(k_{x}\right) \cos \left(k_{y}\right)
$$

where $\delta_{\alpha \beta}$ is the Kronecker delta function. The dominant pairing hence occurs between the iron $3 d$ electrons in the same orbital and on the next-nearest neighbour Fe atoms. The three solutions that we find, differ in the sign and amplitude of the coefficient $\Delta_{\alpha}$ for different orbitals.

For general orientation, in Fig. 3 we plot the diagonal components of the gap functions in the orbital space $\left(\Delta_{\alpha \alpha}(\mathbf{k})\right)$ in the first Brillouin zone of the single-iron unit cell. We also plot the diagonal components of the pairing function in the band basis $\left(\Delta_{i i}(\mathbf{k})\right)$ on the Fermi surfaces; but notice that the off-diagonal components $\Delta_{i j}(\mathbf{k})$ are equally large.

In these three competing pairing states, each orbital component $\Delta_{\alpha \alpha}(\mathbf{k})$ changes sign between the zone centre and $\mathbf{k}=(\pi, 0)$; hence it has an $s^{+-}$form ${ }^{6}$. The three states differ by the relative phase of individual orbital components, which leads to different gap structures on the Fermi surfaces and to different global symmetries. When all three $t_{2 \mathrm{~g}}$ orbitals have the same phase $\left(\Delta_{x y}>0, \Delta_{x z}>0, \Delta_{y z}>0\right)$, we recover the conventional $s^{+-}$ state $^{6}$. If the $x z$ orbital has the opposite phase to the $y z$ orbital $\left(\Delta_{x z}=-\Delta_{y z}\right)$, the global symmetry is of $d$-wave type. In this case the $x y$ orbital shows negligible pairing $\left(\Delta_{x y} \approx 0\right)$. Finally, we find a novel type of pairing state in which $x z$ and $y z$ orbitals are in 
phase $\left(\Delta_{x z}>0, \Delta_{y z}>0\right)$, but the $x y$ orbital has the opposite phase $\left(\Delta_{x y}<0\right)$. We call this state the orbital-antiphase $s^{+-}$state.

To compare with the experimentally determined superconducting gaps measured on the Fermi surfaces, we transform the gap function from the orbital basis $\left(\Delta_{\alpha \beta}(\mathbf{k})\right)$ to the band basis $\left(\Delta_{i j}(\mathbf{k}), i, j\right.$ are band indices) and diagonalize the Bogoliubov quasiparticle Hamiltonian in the multi-band Hilbert space, to obtain the superconducting gaps on the Fermi surfaces (Supplementary Methods). Notice that in the orbital-antiphase $s^{+-}$state the diagonal part of the pairing function $\Delta_{i i}(\mathbf{k})$ (plotted in Fig. 3) vanishes at four points on the electron Fermi surface, in close proximity to the two-band touching point. Nevertheless, the Bogoliubov gap remains finite because the off-diagonal components of the pairing strength $\Delta_{i j}(\mathbf{k})$ in the band basis remain finite; hence, the interband pairing removes the nodes on the electron pockets (Methods). Unlike in the weak-coupling theories, these off-diagonal components can even induce pairing in bands which are near (but do not touch or cross) the Fermi level.

In Fig. 4 we show the details of the pairing gaps on the twodimensional (cut at $k_{z}=0$ ) Fermi surfaces of LiFeAs for both the conventional $s^{+-}$and the new orbital-antiphase $s^{+-}$states. Our calculations show that the orbital-antiphase $s^{+-}$state has the largest pairing strength in LiFeAs. Experimentally, it was found ${ }^{4,5}$ that the superconducting gaps have a very unusual variation on the Fermi surfaces. The superconducting gap is largest on the inner and smallest on the outer hole Fermi surfaces (Fig. 4c,e). The outer hole pocket has maximal (minimal) gap at $\varphi=45^{\circ}\left(0^{\circ}\right)$, whereas the two electron pockets have maximal (minimal) gap at $\varphi=0^{\circ}\left(45^{\circ}\right)$; see Fig. 4 d,f.

The variation of the gaps on the hole Fermi surfaces is reproduced very well by both the conventional $s^{+-}$state and the orbitalantiphase $s^{+-}$state. The $a b$ initio two-particle vertex function determined by this method improves on previous random phase approximation calculations ${ }^{23}$, where the superconducting gap was found to be larger on the outer hole Fermi surface as compared to the inner hole Fermi surface, in disagreement with experiment. We note that previous functional renormalization group calculations ${ }^{24}$ predicted the opposite angular variation of the gap on the outer hole Fermi surface.

The experimentally determined gap variation on the electron pockets is more consistent with the orbital-antiphase $s^{+-}$state having a minimum gap at $\varphi=45^{\circ}$ for both electron pockets. In the conventional $s^{+-}$state, the outer electron pocket has a maximum gap at $\varphi=45^{\circ}$, which was used in ref. 4 as evidence against the spinfluctuation mechanism of superconductivity.

Figures $4 \mathrm{a}$,b exhibit a qualitative difference between the orbitalantiphase $s^{+-}$and the conventional $s^{+-}$states: the sign change of the order parameter between inner and outer hole pockets as well as between inner and outer electron pockets. Weak coupling theories with large hybridization between the two electron pockets predicted the existence of a sign change of the pairing function between these two pockets ${ }^{25}$. In our theory, this hybridization is very weak and plays no role-rather, it is the orbital character that is the decisive factor in determining the sign change.

Small momentum transfer impurity scattering is pair-breaking in the orbital-antiphase state, an effect which may have already been observed in ref. 26 on optimally doped $(\mathrm{Ba}, \mathrm{K}) \mathrm{Fe}_{2} \mathrm{As}_{2}$. The novel orbital-antiphase $s^{+-}$state may thus be realized in other iron-based superconductors.

\section{Methods}

Our calculations are performed with an ab initio theoretical method for correlated electron materials, based on a combination of dynamical mean field theory (DMFT) and density functional theory (DFT; ref. 27). This computational method improves on the DFT description of the electronic structure of iron-based superconductors, predicts the correct magnitude of the ordered magnetic moments ${ }^{9}$, and improves the description of electronic spectral functions, Fermi surfaces $^{9,14}$ and charge response functions such as the optical conductivity ${ }^{8}$.
To predict the dynamical magnetic response function across the different families of iron-based materials, and their impact on the superconducting pairing, we compute from $a b$ initio both the one-particle Green's function and the two-particle scattering amplitude (also called the two-particle vertex function) ${ }^{28}$. We studied the paramagnetic phase of all compounds at the same temperature and used the same Coulomb interactions (Hubbard $U$ and Hund's coupling $J$ ) for all materials as in our previous work ${ }^{9}$, which were determined by the GW $\operatorname{method}^{29}$. In ref. 9, the nominal valence of $\mathrm{Fe}$, as needed for double counting correction, was fixed to $3 d^{6}$ across all Fe compounds. To improve agreement with experiment, it is better to use the nominal valence of each compound, as has been done in the present paper-for example, $3 d^{5.5}$ for $\mathrm{Fe}$ in $\mathrm{KFe}_{2} \mathrm{As}_{2}$. This results in a substantially larger mass enhancement (by a factor of two for the $x y$ orbital) in $\mathrm{KFe}_{2} \mathrm{As}_{2}$ compared to the previous study in ref. 9 .

To investigate the instability towards superconductivity, we computed, within the DMFT framework, the complete two-particle scattering amplitude in the particle-particle channel, which is written in terms of the fully irreducible two-particle vertex and the reducible two-particle vertices in the particle-hole channel, and we solved Eliashberg equations in the Bardeen-Cooper-Schrieffer (BCS) low-energy approximation (Supplementary Methods). In the Eliashberg equations, the orbital degrees of freedom play the central role, because the Coulomb interactions, and the two-particle irreducible vertex function in the particle-hole channel, are large between the iron $3 d$ electrons on the same iron site.

The leading eigenstates of the pairing interaction, conventional $s^{+-}$, orbital-antiphase $s^{+-}$and the $d$-wave states, which are described by equation (1), are almost degenerate. This can be understood if the largest term of the pairing interaction strength at low energies, $\sum_{\mathbf{k Q} \alpha \beta \gamma \delta} \Gamma_{\mathbf{k}, \mathbf{k}+\mathbf{Q}}^{\alpha \beta \gamma \delta} c_{\mathbf{k}, \alpha, \uparrow}^{\dagger} c_{-\mathbf{k}, \beta, \downarrow}^{\dagger} c_{-\mathbf{k}-\mathbf{Q}, \gamma, \downarrow} c_{\mathbf{k}+\mathbf{Q}, \delta, \uparrow}$ (where $\alpha, \beta, \gamma$ and $\delta$ are orbital indices, $c^{\dagger}$ and $c$ are the creation and annihilation operators, respectively), is almost diagonal in orbital space-namely,

$$
\Gamma_{\mathbf{k}, \mathbf{k}+\mathbf{Q}}^{\alpha \beta \gamma \delta} \approx \Gamma(\mathbf{k}, \mathbf{Q}) \delta_{\alpha \beta} \delta_{\gamma \delta} \delta_{\alpha \gamma}+\cdots
$$

with the dots indicating smaller terms which lift the degeneracy among the three superconducting solutions. This suggests that superconductivity in different orbitals is almost decoupled, which is a consequence of the orbital blocking mechanism ${ }^{9}$ and Hund's interaction, acting as an orbital decoupler ${ }^{30}$.

The subleading terms in equation (2) couple different orbitals, and determine which superconducting state is realized in a given material. Among the $t_{2 \mathrm{~g}}$ orbitals, the $x y$ orbital plays a special role-it carries most of the magnetic moment ${ }^{8}$, has the largest effective mass ${ }^{9}$, and with increasing Hund's coupling it decouples first from the $x z$ and $y z$ orbital. This decoupling of the $x y$ orbital allows the sign change in the $\Delta_{x y}$ leading to orbital-antiphase superconductivity. As a consequence, the subleading interaction between electrons on the outer and the inner (both electron and hole) pocket is repulsive. The Hund's coupling is thus not only responsible for the emergence of the large fluctuating moment ${ }^{9}$ and sizable correlation strength in Fe-superconductors ${ }^{7}$, but also the symmetry of the pairing function.

To understand the results qualitatively, we examine the coupling of the orbitals in the vertex function using schematic BCS equations. In the conventional $s^{+-}$case, the orbitals are very tightly coupled (for example, $\left.\Gamma_{\mathbf{k}, \mathbf{k}+\mathbf{Q}}^{\alpha \beta \gamma \delta} \approx \Gamma(\mathbf{k}, \mathbf{Q}) \delta_{\alpha \beta} \delta_{\gamma \delta}+\cdots\right)$, hence the eigenvector with the largest eigenvalue $\lambda$ of the BCS equations, written schematically as $\lambda \Delta_{\alpha}=\sum_{\gamma} \Gamma N_{\gamma} \Delta_{\gamma}$, has equal sign in all orbitals $\left(\Delta_{x z}>0, \Delta_{y z}>0, \Delta_{x y}>0\right)$. $\left(N_{\gamma}\right.$ is the partial density of states with $\gamma$ character.) In contrast, the BCS equations with interaction from equation (2) will have the leading term of the form $\lambda \Delta_{\alpha}=\Gamma N_{\alpha} \Delta_{\alpha}$, which is insensitive to the sign of pairing $\Delta_{\alpha}$, and hence all three types of pairings (conventional $s^{+-}$, orbital-antiphase $s^{+-}$and $d$-wave) are exactly degenerate.

Although the structure of the pairing interaction is particularly simple in the orbital basis, the understanding of the excitation spectra requires the transformation to the band basis. In the band basis, it is essential to retain the large off-diagonal matrix elements of $\Delta_{i j}(\mathbf{k})$ when computing the Bogoliubov gap. Near the point of band degeneracy $\left(\varepsilon_{1}(\mathbf{k})=\varepsilon_{2}(\mathbf{k})=0\right)$, the diagonal component of the pairing function $\Delta_{11}$ changes sign in the orbital-antiphase state; however, the Bogoliubov gap remains finite and approximately equal to $\sqrt{\Delta_{11}^{2}+\Delta_{12}^{2}}$, where $\Delta_{12}$ is the interband pairing amplitude, which removes nodes near such degeneracies (Supplementary Methods).

Received 5 November 2013; accepted 2 September 2014; published online 12 October 2014

\section{References}

1. Stewart, G. R. Superconductivity in iron compounds. Rev. Mod. Phys. 83, 1589-1652 (2011).

2. Hirschfeld, P. J., Korshunov, M. M. \& Mazin, I. I. Gap symmetry and structure of Fe-based superconductors. Rep. Prog. Phys. 74, 124508 (2011). 
3. Dai, P., Hu, J. \& Dagotto, E. Magnetism and its microscopic origin in iron-based high-temperature superconductors. Nature Phys. 8, 709-718 (2012).

4. Borisenko, S. V. et al. One-sign order parameter in iron based superconductor. Symmetry 4, 251-264 (2012).

5. Umezawa, K. et al. Unconventional anisotropic s-wave superconducting gaps of the LiFeAs iron-pnictide superconductor. Phys. Rev. Lett. 108, 037002 (2012).

6. Mazin, I. I., Singh, D. J., Johannes, M. D. \& Du, M. H. Unconventional superconductivity with a sign reversal in the order parameter of $\mathrm{LaFeAsO}_{1-x} \mathrm{~F}_{x}$ Phys. Rev. Lett. 101, 057003 (2008).

7. Haule, K. \& Kotliar, G. Coherence-incoherence crossover in the normal state of iron-oxypnictides and importance of the Hund's rule coupling. New J. Phys. 11, 025021 (2009).

8. Yin, Z. P., Haule, K. \& Kotliar, G. Magnetism and charge dynamics in iron pnictides. Nature Phys. 7, 294-297 (2011).

9. Yin, Z. P., Haule, K. \& Kotliar, G. Kinetic frustration and the nature of the magnetic and paramagnetic states in iron pnictides and iron chalcogenides. Nature Mater. 10, 932-935 (2011).

10. Harriger, L. W. et al. Nematic spin fluid in the tetragonal phase of $\mathrm{BaFe}_{2} \mathrm{As}_{2}$. Phys. Rev. B 84, 054544 (2011).

11. Wang, M. et al. Antiferromagnetic spin excitations in single crystals of nonsuperconducting $\mathrm{Li}_{1-x}$ FeAs. Phys. Rev. B 83, 220515(R) (2011).

12. Liu, M. S. et al. Nature of magnetic excitations in superconducting $\mathrm{BaFe}_{1.9} \mathrm{Ni}_{0.1} \mathrm{As}_{2}$. Nature Phys. 8, 376-381 (2012).

13. Wang, M. et al. Doping dependence of spin excitations and its correlations with high-temperature superconductivity in iron pnictides. Nature Commun. 4, 2874 (2013).

14. Yin, Z. P., Haule, K. \& Kotliar, G. Fractional power-law behavior and its origin in iron-chalcogenide and ruthenate superconductors: Insights from first-principles calculations. Phys. Rev. B 86, 195141 (2012).

15. Margadonna, S. et al. Pressure evolution of the low-temperature crystal structure and bonding of the superconductor FeSe $\left(\mathrm{T}_{\mathrm{c}}=37 \mathrm{~K}\right)$. Phys. Rev. B 80, 064506 (2009).

16. Ikeda, H., Arita, R. \& Kuneš, J. Doping dependence of spin fluctuations and electron correlations in iron pnictides. Phys. Rev. B 82, 024508 (2010).

17. Rhee, H. B. \& Pickett, W. E. Contrast of LiFeAs with isostructural, isoelectronic, and non-superconducting MgFeGe. J. Phys. Soc. Jpn 82, 034714 (2013).

18. Jeschke, H. O., Mazin, I. I. \& Valenti, R. Why MgFeGe is not a superconductor Phys. Rev. B 87, 241105(R) (2013).

19. Bao, W. et al. Incommensurate magnetic order in the $\alpha-\mathrm{Fe}(\mathrm{Te}, \mathrm{Se})$ superconductor systems. Phys. Rev. Lett. 102, 247001 (2009).

20. Scalapino, D. J. A common thread: The pairing interaction for unconventional superconductors. Rev. Mod. Phys. 84, 1383-1417 (2012).

21. Lee, C. H. et al. Incommensurate spin fluctuations in hole-overdoped superconductor $\mathrm{KFe}_{2} \mathrm{As}_{2}$. Phys. Rev. Lett. 106, 067003 (2011).
22. Bao, W. et al. A novel large moment antiferromagnetic order in $\mathrm{K}_{0.8} \mathrm{Fe}_{1.6} \mathrm{Se}_{2}$ Superconductor. Chin. Phys. Lett. 28, 086104 (2011).

23. Wang, Y. et al. Superconducting gap in LiFeAs from three-dimensional spin-fluctuation pairing calculations. Phys. Rev. B 88, 174516 (2013).

24. Platt, C., Thomale, R. \& Hanke, W. Superconducting state of the iron pnictide LiFeAs: A combined density-functional and functional-renormalization-group study. Phys. Rev. B 84, 235121 (2011).

25. Khodas, M. \& Chubukov, A. V. Interpocket pairing and gap symmetry in Fe-based superconductors with only electron pockets. Phys. Rev. Lett. 108, 247003 (2012)

26. Zhang, P. et al. Observation of momentum-confined in-gap impurity state in $\mathrm{Ba}_{0.6} \mathrm{~K}_{0.4} \mathrm{Fe}_{2} \mathrm{As}_{2}$ : Evidence for anti-phase $s_{ \pm}$pairing. Phys. Rev. X 4, 031001 (2014)

27. Kotliar, G. et al. Electronic structure calculations with dynamical mean-field theory. Rev. Mod. Phys. 78, 865-951 (2006)

28. Park, H., Haule, K. \& Kotliar, G. Magnetic excitation spectra in $\mathrm{BaFe}_{2} \mathrm{As}_{2}$ : A two-particle approach within a combination of the density functional theory and the dynamical mean-field theory method. Phys. Rev. Lett. 107, 137007 (2011)

29. Kutepov, A., Haule, K., Savrasov, S. Y. \& Kotliar, G. Self consistent GW determination of the interaction strength: Application to the iron arsenide superconductors. Phys. Rev. B 82, 045105 (2010).

30. De' Medici, L., Giovannetti, G. \& Capone, M. Selective Mott physics as a key to iron superconductors. Phys. Rev. Lett. 112, 177001 (2014).

\section{Acknowledgements}

We thank H. Park, P. Dai and H. Ding for stimulating discussions. This work is supported by NSF DMR-1308141 (Z.P.Y. and G.K.) and NSF DMR-1405303 (K.H.). This research used resources of the Oak Ridge Leadership Computing Facility at the Oak Ridge

National Laboratory, which is supported by the Office of Science of the US Department of Energy under Contract No. DE-AC05-00OR22725.

\section{Author contributions}

Z.P.Y. carried out the calculations. K.H. developed the DMFT code. Z.P.Y., K.H. and G.K. analysed the results and wrote the paper. Z.P.Y. led the project.

\section{Additional information}

Supplementary information is available in the online version of the paper. Reprints and permissions information is available online at www.nature.com/reprints. Correspondence and requests for materials should be addressed to Z.P.Y

\section{Competing financial interests}

The authors declare no competing financial interests. 